\title{
The Differential Diagnosis of Parkinson's Disease
}

\author{
Julia C. Greenland • Roger A. Barker
}

John Van Geest Centre for Brain Repair, Department of Clinical

Neurosciences, University of Cambridge, UK

Author for Correspondence: Julia C. Greenland, John van Geest Centre for Brain Repair, E.D. Adrian Building, Forvie Site, Robinson Way, Cambridge, CB2 OPY, UK. E-mail: jcg69@cam.ac.uk

Doi: http://dx.doi.org/10.15586/codonpublications.parkinsonsdisease.2018.ch6

\begin{abstract}
Parkinson's disease (PD) is a common, progressive neurodegenerative disease. It is a movement disorder presenting primarily with a combination of bradykinesia, rigidity and tremor. However, it has a variable presentation, including the presence of non-motor symptoms such as cognitive impairment and sleep disturbance. The diagnosis is made clinically by the recognition of these key features and the exclusion of other causes of parkinsonism. This chapter describes how to recognize the core motor and non-motor features of PD, as well as atypical features that suggest an alternative cause of parkinsonism. The essentials of these alternative diagnoses are outlined, and the list of differentials is structured into sections on other neurodegenerative causes of parkinsonism, secondary causes of parkinsonism, genetic causes of parkinsonism, tremor disorders, and nonneurological differentials of PD.
\end{abstract}

Keywords: Diagnosis; Differential; Movement Disorder; Parkinsonism; Parkinson's Disease

In: Parkinson's Disease: Pathogenesis and Clinical Aspects. Stoker TB, Greenland JC (Editors). Codon Publications, Brisbane, Australia. ISBN: 978-0-9944381-6-4; Doi: http://dx.doi. org/10.15586/codonpublications.parkinsonsdisease.2018

Copyright: The Authors.

Licence: This open access article is licenced under Creative Commons Attribution 4.0 International (CC BY 4.0). https://creativecommons.org/licenses/by-nc/4.0/ 


\section{INTRODUCTION}

Idiopathic Parkinson's disease (PD) is a common, progressive neurodegenerative disorder, affecting approximately $1 \%$ of people over 65 years of age (1). Patients present to health services in a number of settings, and the key to its diagnosis is recognition of its core features, as PD remains a clinical diagnosis with no diagnostic test. A definitive diagnosis of PD is only possible postmortem and relies on the degeneration of the substantia nigra with the presence of Lewy body pathology, the main constituent of which is alpha-synuclein ( $\alpha$-synuclein) (2). Both structural and dopaminergic imaging can be helpful in specific scenarios, but only once the clinical phenotype has been established. PD is traditionally classified as a movement disorder, which is reflected in the diagnostic criteria (see the section below and Table 1) (3). However, the non-motor features are now well established and often precede the onset of the motor syndrome and thus can help in making the true diagnosis (4), although equally the heterogeneity of its presentation and the disease course means that PD can mimic other conditions.

\section{THE DIAGNOSIS OF IDIOPATHIC PARKINSON'S DISEASE}

The first component to the diagnosis of PD is establishing that the patient has "parkinsonism." This is a clinical diagnosis and relies on three key elements: bradykinesia, tremor, and rigidity. Of these, bradykinesia must be present, with at least one of the other two. PD is an asymmetrical condition, so during the clinical assessment, the parkinsonism should be more apparent on one side and may be purely unilateral in early disease (3). Figure 1 is a classical illustration of parkinsonism, with a description by William Gowers.

Once it has been established that a patient has parkinsonism, in order to diagnose PD, it is vital to exclude other causes of this syndrome. This includes looking for atypical features in the history and on examination. Furthermore, in addition to the presence of parkinsonism, there are also additional features which contribute toward a positive clinical diagnosis of $\mathrm{PD}$. The presence of nonmotor features is important, as these may be prominent even early in the disease course. The response to treatment is equally supportive, as is the evolution of the clinical syndrome over time. These features will be discussed more thoroughly in the sections below, and they are summarized in the clinical diagnostic criteria in Table 1 as exclusion criteria, supportive criteria, and "red flags" (3).

\section{Bradykinesia}

Bradykinesia is a slowness and lack of spontaneous movement. Patients may report difficulty with fine motor tasks, such as doing-up buttons; a change in speech with a quiet, monotonous voice (hypophonia); or an increase in saliva because of an infrequent swallow. Even before examining a patient, bradykinesia can be appreciated by noticing a lack of normal gestures and fidgeting, the loss of facial expression (hypomimia), and a decreased blink frequency. It is helpful to observe their gait as they walk into the clinic room; they may exhibit a reduced arm swing as well as short, shuffling steps and a stooped posture. 


\section{TABLE 1 The clinical diagnostic criteria for Parkinson's disease, based on the Movement Disorder Society guidelines}

1. Diagnosis of parkinsonism
a. Bradykinesia Plus one of
b. Tremor
c. Rigidity

2. Exclusion criteria
a. Cerebellar abnormalities
b. Supranuclear gaze palsy
c. Diagnosis of behavioral variant of frontotemporal dementia or primary progressive aphasia within 5 years of disease onset
d. Parkinsonian features restricted to the lower limbs for more than 3 years
e. Treatment with a dopamine receptor blocker or dopamine depleting agent consistent with drug- induced parkinsonism
f. Absence of a response to high-dose levodopa despite at least moderate disease severity
g. Cortical sensory loss, clear limb ideomotor apraxia, or progressive aphasia
h. Normal functional imaging of the dopaminergic system ("DAT scan")
i. Diagnosis of alternative condition causing parkinsonism which could be causing the symptoms

3. Supportive criteria
a. Clear beneficial response to dopaminergic therapy
b. Presence of levodopa-induced dyskinesia
c. Rest tremor of a limb
d. The presence of either olfactory loss or cardiac sympathetic denervation on MIBG scintigraphy (although the latter is rarely done in current practice)

4. Red flags
a. Rapid progression of gait impairment leading to wheelchair use within 5 years
b. Absence of progression of motor symptoms over 5 years, unless related to treatment
c. Early bulbar dysfunction
d. Inspiratory respiratory dysfunction
e. Severe autonomic failure within the first 5 years of disease
f. Recurrent falls because of impaired balance within 3 years of onset
g. Disproportionate anterocollis or contractures within 10 years of disease onset
h. Absence of any of the common non-motor features despite 5 years of disease
i. Unexplained pyramidal signs
j. Bilateral symmetrical parkinsonism

For the diagnosis of clinically established PD

For the diagnosis of clinically probable PD

1. Parkinsonism

2. Absence of exclusion criteria

1. Parkinsonism

3. At least 2 supportive criteria

2. Absence of exclusion criteria

3. Balanced numbers of supportive criteria and red flags

Bradykinesia can be specifically elicited on examination using repeated hand movements, for example, finger tapping or pronation/supination, or in the lower limbs using repeated foot tapping. Patients with parkinsonian bradykinesia should show not only a slowness of movement but also a decrement in the speed or amplitude of movement (5). A change in handwriting is another manifestation of bradykinesia which can be demonstrated during the clinical examination; patients 

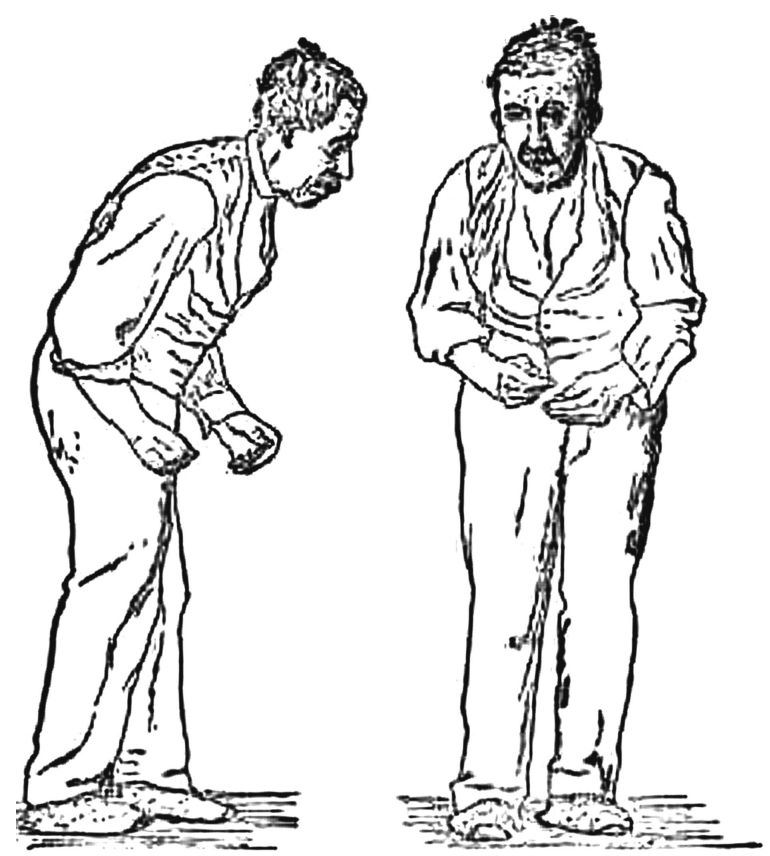

Figure 1 A case of Parkinson's disease as described and illustrated by William Gowers: "... the aspect of the patient is very characteristic. The head is bent forward, and the expression of the face is anxious and fixed, unchanged by any play of emotion. The arms are slightly flexed at all joints from muscular rigidity, and (the hands especially) are in constant rhythmical movement, which continues when the limbs are at rest so far as the will is concerned. The tremor is usually more marked on one side than on the other. Voluntary movements are performed slowly and with little power. The patient often walks with short quick steps, leaning forward as if about to run (61)."

display small and cramped handwriting which becomes progressively smaller (micrographia) with a tendency of the sentence to fall off the line.

On occasion, the true bradykinesia of PD can be mistaken because the patient has another comorbidity leading to difficulty in repeated hand movements, for example, psychomotor slowness in depression, the pain of arthritis, or other neurological disease including cerebellar ataxia or dyspraxia. In ambiguous cases, it is therefore important to rely on other clues in the examination and assess the patient for global bradykinesia (6).

\section{Tremor}

A parkinsonian tremor is classically described as "pill-rolling" (pronation/supination) and low frequency $(4-6 \mathrm{~Hz}$ ). It should start unilaterally but can progress to involve both sides, although it should remain asymmetric. It is apparent in the most distal part of a limb, that is, the hand if the upper limb is involved. It should typically occur at rest and improve or disappear on action. In early stage disease, it is typically intermittent and patients often notice it while relaxing, for example, watching television, and consequently, it is useful to watch for the tremor during the entirety of the consultation. The tremor also becomes more prominent when the patient is 
engaged in another task, such as counting backwards or walking. The parkinsonian tremor also often shows a re-emergence phenomenon whereby it disappears following an action, but then after a few moments becomes apparent again (7). It is seen most commonly in the upper limbs, including just a finger or thumb, but can also involve the legs, chin, or jaw. Although tremor is often identified as a key feature of $\mathrm{PD}$, by health professionals and members of the public alike, it does not need to be present for a diagnosis of $\mathrm{PD}$, and a proportion will never have a tremor $(5,8)$. One study reported that $69 \%$ had a rest tremor at disease onset, with $75 \%$ having a tremor at some point in their disease course (9).

\section{Rigidity}

Patients will often report unilateral pain or stiffness, commonly in the shoulder. This can represent the first presentation of PD, leading to referrals to rheumatologists or orthopedic surgeons, and initial diagnoses of frozen shoulder (10).

Rigidity needs to be appreciated on examination, with the patient in a relaxed position. It can be felt through a range of passive movements of a joint (flexion, extension, and rotation). It is often best felt distally at the wrist, and in early disease may only become apparent with a reinforcement manoeuver, whereby the patient is asked to perform an action on the contralateral side (e.g., to raise the arm up and down), although a slight increase in tone with this manoeuver is seen in many people without PD. The increase in tone should be independent of velocity and is described as "lead-pipe" resistance, often with the "cogwheel" phenomenon, where a superimposed tremor is also felt (5).

\section{Non-motor features}

The non-motor features of PD contribute toward the certainty of diagnosis. There is established evidence of a pre-motor phase, which is thought to represent the onset of the neurodegenerative pathology. Patients who go on to develop PD commonly have experienced depression, constipation, anosmia, and REM sleep behavior disorder in the years preceding their diagnosis (11). Of course, these symptoms are non-specific and common in the elderly population, with many potential causes, which complicate their use in predicting those at risk of subsequently developing PD. However, their presence gives support to the diagnosis of $\mathrm{PD}$ and should be specifically queried during the diagnostic consultation.

There are further non-motor aspects to PD which can develop at any point through the disease course. Subtle cognitive deficits can be present even at diagnosis and typically affects attentional, executive, visuospatial, and memory functions (12). Neuropsychiatric symptoms are also common and cover depression, anxiety, and apathy as well as psychosis. Psychosis has a spectrum of presentations from minor illusions, for example, where patients report that they glimpse things out of the corner of their eye that turn out not to be real, through to formed hallucinations and delusions with a lack of insight, leading in some cases to delusional paranoid behavior (13). Autonomic dysfunction can manifest as urinary frequency or urgency, constipation, orthostatic hypotension, drooling, erectile dysfunction, or abnormal sweating (14).

These non-motor features initially present insidiously and non-specifically, and the patient will often think they are unrelated to PD and not volunteer them 
during a consultation. Moreover, they are typically not treated by the dopaminergic therapy which is prescribed primarily for the rigidity and bradykinesia (4). It is therefore important to actively screen for them, for example, checking a lying and standing blood pressure and asking about sleep, as they can have a substantial impact on the patient's quality of life (15), and similar to the motor features of PD, there are many good treatments available.

\section{Disease progression}

Progression is an important diagnostic feature of PD. By the time the patient presents to clinic, there should be a history describing a worsening of symptoms to the point of seeking medical attention. It is then important that at each subsequent review, this story of disease progression is reviewed, because if lacking or abnormally rapid, it may point toward an alternative diagnosis. A caveat to this is that although all cases do worsen, there is a large range in the rates at which this occurs. Ten years following diagnosis, about one-fifth of patients are doing very well and have very few complications associated with the disease, while $40 \%$ will have gone on to develop a dementia (16). Equally, patients who respond well to medication may appear to have many years of stable disease with little worsening of symptoms and this can sometimes be mistaken as a "cure" by the patient.

As the disease progresses, other motor features become apparent which are characteristic of PD. As mentioned above, gait impairment is common. However, this becomes more pronounced and patients adopt a characteristic stooped posture with reduced arm swing (see Figure 1 for a classical illustration of this posture). The arm itself can also adopt an abnormal flexed posture on walking. Classically, patients have a flexed neck and camptocormia, where there is truncal flexion at the thoracolumbar spine. The shuffling steps can progress so that patients display festination whereby successive steps are shorter. Postural instability develops, which has been shown to have a significantly detrimental effect on quality of life, and leads to an increased risk of falls and associated morbidity (15). Patients may progress to develop freezing of gait, which is also very disabling with an associated falls risk. This is where patients are unable to initiate or continue walking and describe a sensation like their feet are stuck to the floor. It commonly occurs on turning, walking through a narrow space (such as a doorway), or on starting to walk (17).

A positive clinical response to levodopa is supportive of the diagnosis of PD, and this is the case particularly for bradykinesia and stiffness. Levodopa has a more variable effect on tremor. Equally supportive are the presence of dyskinesias or additional choreoform involuntary movements, which can develop as the disease progresses in response to the dose and duration of dopaminergic therapy (18).

\section{OTHER NEURODEGENERATIVE CAUSES OF PARKINSONISM}

The following conditions can mimic idiopathic PD, and on occasion, the correct diagnosis only becomes apparent with time. However, there are key features on history and examination to recognize in order to discriminate between these conditions. 


\section{Dementia with Lewy bodies}

Dementia with Lewy bodies (DLB) is the second most common cause of dementia, following Alzheimer's disease. It is characterized by progressive cognitive impairment together with fluctuating levels of attention, visual hallucinations, REM sleep behavior disorder, and parkinsonism. The predominant pathology in both PD and DLB is Lewy bodies, the main constituent of which is $\alpha$-synuclein, and there is undoubtedly a clinical and pathological overlap. Current diagnostic criteria state that if the cognitive symptoms pre-date or develop within the first year of the emergence of parkinsonism, then the diagnosis is DLB. Should cognitive impairment develop in the context of established PD, then the diagnosis is Parkinson's disease dementia (PDD) (19).

The cognitive impairment of DLB affects attentional-executive and visuospatial functions. Patients typically perform poorly on the Stroop and the trail-making tasks as well as drawing tasks, classically clock-drawing or intersecting pentagons (see Figure 2). Fluctuating attention can manifest as inconsistent behavior and periods of "zoning-out", and in fact, the presence of major fluctuations in a patient's cognitive state is a very helpful diagnostic feature of this condition. The visual hallucinations that occur in approximately $80 \%$ of patients are complex and tend to involve humans or animals. Patients have a variable amount of insight into their presence (19).

Other supportive features include a sensitivity to neuroleptic medication, postural instability and falls, autonomic dysfunction, and other neuropsychiatric features that include depression, apathy, anxiety, delusions, and hallucinations of other modalities. Treatment is based on a multi-disciplinary approach (19). Of note, the parkinsonism is less responsive to dopaminergic therapies, which can worsen cognition and behavior. Cholinesterase inhibitors have a role in management of the neuropsychiatric features (20), as can low-dose quetiapine. In all cases, however, when this problem emerges, other reversible causes should be sought, such as an underlying infection or subdural hematoma.

\section{Multiple system atrophy}

Multiple system atrophy (MSA) is another $\alpha$-synucleinopathy, although much rarer than PD (21). There are a range of associated clinical features, which include parkinsonism, cerebellar ataxia, autonomic failure, urogenital dysfunction, and corticospinal involvement. There is a relative preservation of cognition. It is subdivided

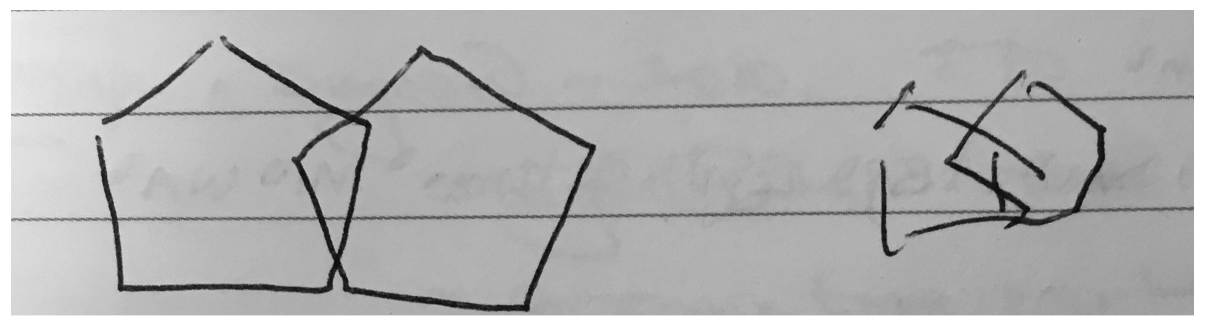

Figure 2 An illustration of the difficulties exhibited by patients with DLB when performing the interlocking pentagon copying task. 
into MSA with predominant parkinsonism (MSA-P) and MSA with predominant cerebellar ataxia (MSA-C).

The parkinsonism of MSA tends to be rapidly progressive and poorly responsive to levodopa. Bradykinesia and rigidity are usually predominant; a classic pillrolling tremor is rarely seen. In the initial stages, MSA can be difficult to distinguish from PD, as symptoms are invariably asymmetric and up to 30\% of patients do show an initial good response to levodopa treatment. MSA patients treated with levodopa can also exhibit dyskinesias, although these are often atypical and can manifest as prolonged facial dystonia or torticollis (22).

Autonomic failure is virtually always a feature at presentation, which tends to occur later in the disease course in PD. This is seen as an orthostatic drop in blood pressure, urinary incontinence, or erectile dysfunction. Additional features which point toward MSA and are not seen in PD are stridor, a positive Babinski sign, and cerebellar ataxia, which can manifest as a gait or limb ataxia, dysarthria, or oculomotor dysfunction. Myoclonus is also seen, particularly as "poly-mini-myoclonus": stretch-sensitive jerks affecting the fingers. This is unusual in PD $(22,23)$.

Imaging can be useful in discriminating PD and MSA. Structural imaging using MRI can reveal changes in the brainstem and basal ganglia which are supportive of MSA, including the "hot cross bun" sign, although this feature is only normally recognized by radiologists familiar with these types of syndrome (23).

Overall, the clue that the patient has MSA rather than PD is the poor response to levodopa and the involvement of systems outside the striatum and thus the prominent gait and autonomic problems early on in the disease course.

\section{Progressive supranuclear palsy}

This neurodegenerative disorder is a tauopathy which usually has a distinct presentation from PD. Patients usually present in their 60s with walking difficulties, unsteadiness, falls, and visual symptoms-often dry eyes and blurred vision (24). The parkinsonism of PSP tends to be symmetrical, involves the axial musculature, and is poorly responsive to levodopa therapy. This axial rigidity means that PSP patients tend to have a very different posture to classically stooped PD patients; they are upright, occasionally with retrocollis. Although postural instability occurs in PD and is a key marker of disease progression, in PSP it can be early and pronounced. Patients fall frequently, often backwards, especially when turning. It is tested clinically by the pull test. Equally, freezing of gait can be prominent and poorly responsive to levodopa (25).

Eye movements in PSP are abnormal. Early in the disease, this can be subtle and manifest only as square wave jerks or slowing of vertical saccades. However, as it progresses, patients develop a restriction of vertical eye movements, which can be overcome with activation of the vestibulo-occular reflex. However, it should be noted that a slight reduction in upgaze is common in the elderly with no underlying pathology in the central nervous system (25).

PSP is associated with a behavioral and cognitive syndrome, which can be apparent at the time of initial presentation. Apathy is characteristic, occurring in $80 \%$ of patients. Paradoxically, so is impulsivity. The most common cognitive deficits are in executive function and verbal fluency (26).

Similar to MSA, neuroimaging can be helpful, with mid-brain atrophy and the "hummingbird sign," (where the atrophied midbrain viewed in the sagittal 
plane resembles the head and beak of a hummingbird) being supportive of the diagnosis (27).

Overall, the clue that the patient has PSP rather than PD is a poor response to levodopa, early falls, eye movement abnormalities, and rapid progression.

\section{Corticobasal syndrome}

Corticobasal syndrome (CBS) is another tauopathy which is both clinically and pathologically heterogenous. As well as a classical CBS presentation, there are three further clinical phenotypes all associated with corticobasal degeneration pathology: a frontal behavioral-spatial syndrome, a non-fluent primary progressive aphasia, and a PSP syndrome (28).

The diagnosis of CBS is clinical and based primarily on its motor features. It causes asymmetric limb rigidity or akinesia, which may resemble the parkinsonism of PD. However, this is frequently associated with dystonia and myoclonus, and the parkinsonism is typically levodopa-resistant and may also involve the axial musculature. Tremor may also be a feature, although it is not the typical rest tremor of PD (28).

Furthermore, there are multiple higher cortical features, which are key in distinguishing this condition from PD. These include apraxia, cortical sensory loss, progressive non-fluent aphasia, and alien limb phenomenon, where the patients have the feeling that the limb does not belong to them; has a will of its own; or independently performs complex, unintentional tasks. Cognitive impairment is heterogenous and has been shown to affect a range of modalities including executive function, episodic memory, visuospatial function, and cognitive flexibility. Behavioral changes may also be prominent and include apathy, irritability, antisocial behavior, personality changes, and hypersexuality (28).

Imaging may be helpful, typically showing an asymmetric pattern of atrophy primarily involving the frontal and parietal cortices. However, it is not diagnostic (29).

\section{SECONDARY CAUSES OF PARKINSONISM}

A number of secondary causes of parkinsonism need to be considered before making the diagnosis of idiopathic PD.

\section{Drug-induced parkinsonism}

Drugs that can act on dopamine receptors in the central nervous system can cause parkinsonism, as well as other movement disorders including tardive dyskinesia, akathisia, and dystonia. Antipsychotics are the most common cause of druginduced parkinsonism, particularly the "typical antipsychotics" including haloperidol and chlorpromazine, although these drugs are now rarely used in clinical practice. However, atypical antipsychotics such as risperidone and olanzapine can also cause extra-pyramidal side effects, including parkinsonism.

Other causes of drug-induced parkinsonism include anti-emetics (e.g., metoclopramide and domperidone), calcium channel blockers (e.g., flunarizine and cinnarizine), anti-epileptics (e.g., sodium valproate and phenytoin), dopamine 
depleting drugs (e.g., tetrabenazine), and selective serotonin reuptake inhibitor anti-depressants (30).

Drug-induced parkinsonism is said to typically cause a symmetrical parkinsonism syndrome with prominent bradykinesia and rigidity; however, this is of course not always the case, and it can closely mimic idiopathic PD. The symptoms typically resolve within weeks to months after withdrawal of the offending drug. However, there is also a proportion of patients in whom the drug has unmasked early underlying PD; in these cases, the parkinsonism will progress despite cessation of the drug. In cases where there is doubt over the diagnosis, a DaTSCAN is useful, as it is normal in drug-induced parkinsonism (30).

\section{Toxins}

A causative link between PD and exposure to toxins was first revealed in the 1980s in California when intravenous drug-users began presenting with acute parkinsonism, after injecting what was found out to be MPTP, a contaminant of their pethidine analog. This knowledge has since been used in research to create animal models of PD (31).

Epidemiological studies have gone on to reveal associations between environmental toxin exposure and the risk of developing PD. These include pesticides (rotenone, paraquat, organophosphates), solvents, and a limited amount of evidence for metals (lead, manganese, mercury). It seems that in these cases, environmental exposure is one of many potential etiological factors, but only explains the development of the disease in part (31). In these cases, MRI scans of the brain are often abnormal with signal change in the basal ganglia (32).

Acute carbon monoxide poisoning has been shown to cause parkinsonism, usually developing within 1 month of exposure. The clinical features can include bradykinesia, rigidity, hypomimia, and gait disturbance, but this is in the context of encephalopathy and frequent frontal lobe signs such as the grasp reflex. Prognosis is good, with most patients making a spontaneous full recovery (33).

\section{Vascular parkinsonism}

Parkinsonism caused by cerebrovascular disease is an important differential to consider in the elderly because it is common, accounting for an estimated 3-6\% of cases of parkinsonism, and has a different prognosis and response to treatment. It is associated with increasing age and vascular risk factors, such as hypertension, previous TIAs, and diabetes mellitus. The syndrome is caused by a lesion affecting either the substantia nigra or its projections, which can include subcortical white matter disease or lacunar infarcts $(34,35)$.

Vascular parkinsonism has a distinct clinical phenotype. It tends to be bilateral, symmetrical and affects the lower limbs, so causing a predominant gait disorder. The gait is typically upright with a broad base, short steps, and normal arm-swing, compared to the stooped posture, narrow base, and reduced arm-swing of PD. Upper limbs are often spared, tremor is rare, and increased tone tends to be a combination of spasticity and rigidity, with gegenhalten paratonia. The clue is that the examination on the bed is relatively normal compared to the problems seen while mobilizing. Progression is typically stepwise and rapid. There are also often 
supporting features, such as the presence of an extensor plantar response, brisk reflexes, a pseudobulbar palsy, and cognitive impairment on examination $(34,35)$.

Both PD and cerebrovascular disease are common in the elderly, so there can be an overlap, causing diagnostic difficulty. Vascular disease on brain imaging is supportive, but not conclusive, as these changes can also be present in patients with PD. DaTSCAN can be helpful, as it is usually normal in vascular parkinsonism. However, an abnormal scan could be caused by a focal basal ganglia infarct. Furthermore, although patients with vascular parkinsonism are said not to respond to levodopa therapy, there are a subset of patients who will get some therapeutic benefit. It is therefore recommended that all patients with suspected vascular parkinsonism receive a trial of levodopa. Otherwise, the management is aimed at rehabilitation and controlling vascular risk factors (34).

\section{Normal-pressure hydrocephalus}

Normal-pressure hydrocephalus (NPH) is diagnosed by the presence of a triad of clinical features (gait disorder, incontinence, and cognitive impairment or dementia) together with characteristic radiological appearances of enlarged ventricles. As well as this clinical triad, which can mimic PD, NPH is also associated with parkinsonism in approximately $70 \%$ of cases. Bradykinesia is the most common parkinsonian feature, but rigidity and postural instability are also frequently present. Furthermore, like PD, it is a slowly progressive disorder of the elderly $(36,37)$.

The gait disorder can appear very parkinsonian, with small, shuffling steps, difficulty turning, and falls. Urinary symptoms, which form part of the diagnostic criteria for NPH, are also common in PD. Furthermore, the cognitive impairment in NPH is similar to what is seen in PD, with associated psychomotor slowing, daytime somnolence, and apathy (36).

Structural brain is therefore important if there is an indication that the underlying cause of the parkinsonism is NPH, particularly as this is a treatable condition, with approximately $80 \%$ of patients experiencing an improvement in symptoms following surgical treatment with a CSF shunt (38). However, distinguishing between NPH and atrophy on a CT or MRI brain scan can be difficult, and if in doubt advice from a neurosurgical team familiar with NPH and CSF infusion studies should be sought.

\section{GENETIC CAUSES OF PARKINSONISM}

It is likely to be worthwhile considering genetic investigations in patients who present at a young age, less than 40, and who have at least one affected relative. There are also a number of atypical features that can give a clue regarding the underlying diagnosis.

\section{Familial PD}

Mutations in a number of genes have been found to be associated with familial PD, and although they can look phenotypically different from idiopathic PD, 
some are identical to PD in terms of their presentation, for example, LRRK2. Some of the most well documented are outlined here.

The first gene identified was the SNCA gene, with mutations being linked to autosomal dominant early-onset parkinsonism. The clinical spectrum is broad, from classical PD to an aggressive syndrome with prominent cognitive impairment and autonomic instability which may mimic MSA. Atypical features of myoclonus and hypoventilation can also be present. A severe phenotype is associated with SNCA triplications, whereas duplications have a tendency to present as typical PD and are more common $(39,40)$.

Mutations in the LRRK2 gene are another, more common, cause of autosomal dominant PD. These can lead to a typical clinical picture of PD, although with a broad range of onset age and a less severe clinical phenotype. The mutation is frequent in people of south European, north African, or Ashkenazi Jewish descent $(39,41)$.

Mutations in parkin, PINK1, and DJ-1 are all associated with early-onset parkinsonism with autosomal recessive inheritance. They have a slowly progressive course, with a clinical picture which is typical of $\mathrm{PD}$, and usually respond well to levodopa. Of these, mutations in parkin are most common and the clue to patients having this is their early onset, leg-dominant features, and a slow progression over decades $(39,40)$.

\section{Wilson's disease}

Wilson's disease is a rare autosomal recessive disease caused by a mutation in the ATPB7 gene causing a toxic accumulation of copper. It typically presents in early adulthood, although can manifest up to the fifth or sixth decade of life. It has hepatic, psychiatric, and neurological manifestations. A primary neurological presentation has been reported to occur in up to $68 \%$ of patients and can involve a combination of dysarthria, tremor, dystonia, and parkinsonism. As the disease progresses, patients can subsequently develop choreoathetosis, ataxia, myoclonus, seizures, and eye movement abnormalities.

The parkinsonism of Wilson's disease has been shown to closely mimic idiopathic PD, with a typically asymmetric syndrome consisting of bradykinesia and rigidity. Rest tremor is less common, but can occur. However, the parkinsonian features rarely occur in isolation, without other neurological signs. Furthermore, patients may also have hepatic and psychiatric involvement, with subclinical liver disease being common in a primary neurological presentation. Diagnostic tests include initial copper studies; slit lamp examination for Kayser-Fleischer rings, which are invariably present in patients presenting with a neurological syndrome; and consideration of genetic testing. This is an important differential to consider, because there are effective treatments available (42).

\section{Huntington's disease}

Huntington's disease is a trinucelotide repeat disorder with autosomal dominant inheritance. Typically, the disease presents in middle age as a movement disorder, classically chorea, together with cognitive and behavioral features. However, parkinsonism is also a recognized feature, which can be present when the disease clinically manifests. Also, a longer triplet repeat is associated with a younger and 
distinct phenotype, which is more akin to PD. This juvenile form of the disease, the Westphal variant, presents with a parkinsonian hypokinetic rigid syndrome usually before the age of 20 , but unlike PD the patients typically also have a number of cognitive and behavioral problems $(43,44)$.

\section{Spinocerebellar ataxia}

Spinocerebellar ataxia (SCA) is a progressive disorder causing degeneration primarily in the cerebellum, leading to a predominantly ataxic syndrome. It has a large range of subtypes, with heterogenous clinical syndromes. Parkinsonism has been particularly reported in three of these: SCA2, SCA3, and SCA17, which can also appear to mimic MSA. These are all trinucleotide repeat disorders, inherited in an autosomal dominant fashion. Of these, SCA2 is the most common disorder associated with parkinsonism, which can be the presenting feature and mimic idiopathic PD, including responsiveness to levodopa. However, classical SCA2 manifests as cerebellar ataxia and peripheral neuropathy, and even in a parkinsonian presentation, ataxia tends to develop within a couple of years (45).

SCA3 is divided into a number of subtypes, of which type IV causes predominant parkinsonism, which is levodopa responsive. The other types are characterized by a combination of cerebellar ataxia and pyramidal signs with extrapyramidal rigidity and dystonia or a peripheral neuropathy. However, patients with type IV have also been shown to commonly have additional clinical features as well as parkinsonism, which would be atypical for idiopathic PD. These include eye movement abnormalities and peripheral neuropathy $(45,46)$.

As with SCA2 and SCA3, only a subgroup of patients with SCA17 present with predominant parkinsonism. But in those cases, it can be clinically typical, levodopa responsive PD. Other features of SCA17, which are more classical, include cerebellar ataxia, epilepsy, dementia, psychosis, chorea, and dystonia (45).

\section{Fragile $\mathrm{X}$-associated tremor/ataxia syndrome}

Fragile X-associated tremor/ataxia syndrome (FXTAS) is a neurodegenerative disorder characterized by cerebellar ataxia and intention tremor. It is caused by a premutation CGG trinucleotide repeat expansion of the fragile-X mental retardation 1 (FMR1) gene. The full mutation with $>200$ repeats is associated with intellectual disability with social and behavioral difficulties, usually from adolescence. The premutation, causing FXTAS, is 55-200 repeats, usually presents in middle age in people with normal intelligence, and demonstrates typical imaging findings, which include increased T2 signal in the middle cerebellar peduncle. As well as the cerebellar ataxia, it can also present with parkinsonism, with bradykinesia, postural instability, and rest tremor all being described. However, given the cerebellar features on examination, FXTAS is more commonly mistaken for MSA rather than $\mathrm{PD}(47)$.

\section{Frontotemporal dementia with parkinsonism}

Parkinsonism in frontotemporal dementia (FTD) is usually seen in the behavioral variant, rather than in association with primary progressive aphasia, and can develop either before or during the development of the classical FTD syndrome. 
It can closely mimic idiopathic PD or have features suggestive of PSP or CBS. It is seen in association with underlying tau, TDP-43, or FUS pathology, as well as corresponding mutations in several genes, which include MAPT, PGRN, C9ORF72, FUS, and TARDBP. Rigidity and bradykinesia tend to be the more prominent parkinsonian features, with rest tremor occurring rarely. There is variable responsiveness to levodopa (48).

\section{Neurodegeneration with brain iron accumulation}

Neurodegeneration with brain iron accumulation (NBIA) patients present with a progressive extrapyramidal syndrome associated with iron deposition in the basal ganglia. The two main syndromes are outlined here, although there are additional syndromes including neuroferritinopathy and aceruloplasminemia. The most common of the NBIA disorders is pantothenate kinase-associated neurodegeneration (PKAN), resulting from mutations on the PANK2 gene, accounting for $50 \%$. The classic syndrome manifests in early childhood with a combination of pyramidal (spasticity, hyperreflexia) and extrapyramidal features (dystonia, parkinsonism). PKAN can also rarely present in early adulthood. There are typical MRI findings, with a central hyperintensity with surrounding low signal on T2 images in the globus pallidus, giving the so-called eye-of-the-tiger sign (49).

The second main type of NBIA is PLA2G6-associated neurodegeneration (PLAN). When onset occurs in infancy, PLAN causes progressive motor and mental retardation with cerebellar ataxia, seizures, and pyramidal signs. However, onset can occur later in life which leads to an atypical syndrome that may mimic PD, with rest tremor, rigidity, and bradykinesia and a good response to levodopa. However, patients also exhibit additional features including eye-movement abnormalities and pyramidal signs (49).

\section{Mitochondrial disorders}

Mutations in the POLG gene have also been linked with early onset parkinsonism (50). This gene is implicated in the synthesis and repair of mitochondrial DNA, and a large number of clinical phenotypes are associated with its mutations. These include progressive external ophthalmoplegia, ataxia, and peripheral neuropathy. However, parkinsonism can also occur with bradykinesia and rigidity. This has been described as atypical for idiopathic PD, involving a symmetrical distribution with a postural (not rest) tremor and a poor response to levodopa (51).

\section{Idiopathic basal ganglia calcification}

This is a heterogenous disease associated with mineral deposition in the basal ganglia, as well as in other brain structures. There is a strong familial component, with causative mutations identified in SCL20A2 and PDGFRB. Patients commonly have a movement disorder, with parkinsonian features of akinesia and rigidity which show a variable response to levodopa. Other features include cognitive impairment, gait disorder, pyramidal signs, and a psychiatric presentation. Imaging is crucial in diagnosis to identify the areas of calcification, with CT imaging being more useful than MRI (52). 


\section{TREMOR DISORDERS}

Tremor remains the most well-known feature of PD, among both health professionals and the general public. This is despite the fact that a proportion of patients with PD will never develop a tremor. In some, however, it is the first reported symptom; therefore, it is important to distinguish the resting tremor of PD from other causes of tremor.

\section{Essential tremor}

Essential tremor (ET) is common, with an increasing incidence with age, although it may develop at any age from childhood. It typically progresses slowly until later in life. The neuropathological basis of this tremor is unknown, although there is a strong familial component. People with ET often don't present to a clinician unless there is also a concern about PD or the tremor is very disabling (53).

This tremor is typically present in both upper limbs and occurs on action, either while holding a sustained posture or while performing a voluntary movement. Patients commonly notice the tremor while performing actions such as writing or holding a cup. The tremor may improve with small amounts of alcohol and, as with all tremors, it gets worse with anxiety, illness, or tiredness. Tremor can also occur in other locations, such as the lower limbs, head, and it can affect the voice. For diagnosis, there should be at least a 3-year history and no other neurological signs. There is evidence that ET is a more heterogenous syndrome, including the presence of soft neurological signs in some patients; yet, currently there is no consensus regarding the inclusion of these signs in the diagnosis (8).

ET is easy to differentiate from the classical rest tremor of PD; however, some patients with PD also have a postural-action tremor. It is also important to note that cogwheeling rigidity is common with any tremor syndrome, including ET. Furthermore, a re-emergent tremor is also a feature of $\mathrm{PD}$, which is visible several seconds following maintaining a posture (7). In cases where there is difficulty in distinguishing ET from PD, particularly in tremor-dominant, slowly progressive forms, a DaTSCAN can be useful, as it is normal in ET.

\section{Dystonic tremor}

Another common cause of tremor presenting to neurology services is dystonic tremor. This is a tremor occurring in a part of the body affected by dystonia. Therefore, when assessing someone with tremor, it is key to look for the presence of an abnormal posture, which may be subtle. The tremor is caused by rhythmic muscle contractions, often inconstant and described as "jerky." It is exacerbated by attempts to maintain a normal posture. For example, dystonic tremor commonly occurs with cervical dystonia, causing a dystonic head tremor, which may include upper limb involvement. Another clue to the presence of dystonia is looking for a "geste antagoniste," a voluntary movement that corrects the dystonic posture $(8,54)$.

Where there is diagnostic doubt, as with ET, DaTSCAN is normal. The treatment of choice is typically with local Botox injection. 


\section{NON-NEUROLOGICAL DIFFERENTIALS OF PARKINSON'S DISEASE}

As described at the beginning of the chapter, PD is a heterogenous condition, with a range of non-motor features as well as the motor syndrome of parkinsonism. It has been shown that in approximately $20 \%$ of patients, the non-motor symptom is the presenting feature (55). With this range of potential symptoms, PD also commonly presents to specialties other than neurology, notably rheumatology and psychiatry.

\section{Arthritis}

Pain has been shown to be one of the most common non-motor symptom at presentation in PD, and so, a patient's initial referral is often to a rheumatologist or orthopedic surgeon. Pain is usually related to the affected motor side and, together with the associated rigidity, can resemble a stiff joint. Frozen shoulder, degenerative spinal disease, and osteoarthritis are some of the most common initial diagnoses in cases that later are revised to be PD. These misdiagnoses have also been shown to be related to unnecessary procedures, including steroid injections and spinal surgery $(55,56)$.

Furthermore, rheumatological disease can also resemble PD, and it is possible for someone with a primary underlying polyarthropathy to be mistaken as having PD. Joint stiffness can mimic the rigidity and can make it difficult during clinical examination to distinguish it from the bradykinesia of PD. To help to separate these conditions, it is key to look for decrement on the examination of bradykinesia, which is specific for PD, and to explore other supportive features and disease progression in the history. Finally, it is also important to remember that PD patients can develop musculoskeletal disorders secondary to parkinsonian bradykinesia and rigidity (57).

\section{Depression}

Depression is common in PD, affecting up to an estimated $40 \%$ patients, and can pre-date the motor syndrome (4). The two conditions therefore commonly coexist, but equally they can be mistaken for each other. The psychomotor slowing in depression can mimic the bradykinesia of PD and vice versa. For example, the lack of facial expression and flat affect in depression can be confused with the hypomimia of PD. They also share multiple comorbid features; both depression and PD are associated with sleep disturbance, anxiety, fatigue, apathy, and poor concentration (58).

\section{Obsessional slowness}

This is a rare motor presentation of obsessive-compulsive disorder, which can mimic PD. The motor slowness of obsessional slowness (OS) is not related to the obsessions and compulsive rituals of OCD, but is instead a separate phenomenon, although 
occurring in patients who commonly have the classic features of OCD. Patients exhibit slowness of movement, poor speech production and have difficulty in initiating voluntary actions. However, the motor slowness does not display the decrement during repetitive actions which is seen in PD, and DATScan is normal. Motor speed can equally appear normal for reflexive actions or in times of stress (59).

\section{Psychogenic parkinsonism}

Like other psychogenic movement disorders, the etiology of psychogenic parkinsonism (PP) is presumed to be a conversion or somatoform disorder, without conscious awareness or control. It can occur, but not exclusively, in the presence of other psychological or psychiatric disorders, and stress plays a role in most by precipitating, maintaining, and exacerbating symptoms.

A positive diagnosis is made by the identification of clinical features. The slowness of PP is effortful, without decrement, and improves with distraction, as does the rigidity, which is felt as an active resistance against passive movement. The tremor has a number of features which are not typical of PD; it tends to start abruptly; it has a variable frequency, direction, and distribution; and it also improves with distraction. Testing for postural stability may be helpful, as patients commonly have bizarre or exaggerated responses, without falling. Finally, there may be other clinical features of functional neurological disorders, such as "giveway" weakness, a positive Hoover's sign, or non-anatomical sensory loss (60).

\section{CONCLUSION}

Parkinson's disease is a clinical diagnosis, the accuracy of which, as in much of neurology, relies on a detailed history and thorough examination. The certainty of this diagnosis improves following a positive response to treatment and a clinical picture of steady symptomatic decline when the patient is seen in subsequent appointments. The differential diagnosis of PD outlined here is broad, with many rare causes of parkinsonism. It is certainly not appropriate to investigate all cases of PD, but it is critical to keep an open mind regarding unusual features which may lead you to consider one of these differentials.

Acknowledgment: R.A.Barker is supported by the Wellcome-MRC Cambridge Stem Cell Institute and an NIHR Senior Investigator. Both authors are supported by the NIHR Cambridge Biomedical Research Centre (Cambridge University Hospitals NHS Trust/University of Cambridge). The funding bodies had no role in the writing of this manuscript.

Conflict of interest: The authors declare no potential conflicts of interest with respect to research, authorship and/or publication of this article.

Copyright and permission statement: To the best of our knowledge, the materials included in this chapter do not violate copyright laws. All original sources have been appropriately acknowledged and/or referenced. Where relevant, appropriate permissions have been obtained from the original copyright holder(s). 


\section{REFERENCES}

1. Wirdefeldt K, Adami HO, Cole P, Trichopoulos D, Mandel J. Epidemiology and etiology of Parkinson's disease: A review of the evidence. Eur J Epidemiol. 2011;26 Suppl 1:S1-58. http://dx.doi.org/10.1007/ s10654-011-9581-6

2. Braak H, Del Tredici K, Rub U, de Vos RA, Jansen Steur EN, Braak E. Staging of brain pathology related to sporadic Parkinson's disease. Neurobiol Aging. 2003;24(2):197-211. http://dx.doi.org/10.1016/ S0197-4580(02)00065-9

3. Postuma RB, Berg D, Stern M, Poewe W, Olanow CW, Oertel W, et al. MDS clinical diagnostic criteria for Parkinson's disease. Mov Disord: Official J Mov Disord Soc. 2015;30(12):1591-601. http://dx.doi. org/10.1002/mds.26424

4. Chaudhuri KR, Healy DG, Schapira AH. Non-motor symptoms of Parkinson's disease: Diagnosis and management. Lancet Neurol. 2006;5(3):235-45. http://dx.doi.org/10.1016/S1474-4422(06) 70373-8

5. Jankovic J. Parkinson's disease: Clinical features and diagnosis. J Neurol, Neurosurg \& Psychiatr. 2008;79(4):368-76. http://dx.doi.org/10.1136/jnnp.2007.131045

6. Ali K, Morris HR. Parkinson's disease: Chameleons and mimics. Pract Neurol. 2015;15(1):14-25. http://dx.doi.org/10.1136/practneurol-2014-000849

7. Jankovic J, Schwartz KS, Ondo W. Re-emergent tremor of Parkinson's disease. J Neurol, Neurosurg, Psychiatr. 1999;67(5):646-50. http://dx.doi.org/10.1136/jnnp.67.5.646

8. Bhatia KP, Bain P, Bajaj N, Elble RJ, Hallett M, Louis ED, et al. Consensus statement on the classification of tremors. From the task force on tremor of the International Parkinson and Movement Disorder Society. Mov Disord: Official J Mov Disord Soc. 2018;33(1):75-87. http://dx.doi.org/10.1002/ mds. 27121

9. Hughes AJ, Daniel SE, Blankson S, Lees AJ. A clinicopathologic study of 100 cases of Parkinson's disease. Archiv Neurol. 1993;50(2):140-8. http://dx.doi.org/10.1001/archneur.1993.00540020018011

10. Riley D, Lang AE, Blair RD, Birnbaum A, Reid B. Frozen shoulder and other shoulder disturbances in Parkinson's disease. J Neurol, Neurosurg \& Psychiatr. 1989;52(1):63-6. http://dx.doi.org/10.1136/ jnnp. 52.1 .63

11. Tolosa E, Gaig C, Santamaria J, Compta Y. Diagnosis and the premotor phase of Parkinson disease. Neurology. 2009;72(7 Suppl):S12-20. http://dx.doi.org/10.1212/WNL.0b013e318198dbll

12. Muslimovic D, Post B, Speelman JD, Schmand B. Cognitive profile of patients with newly diagnosed Parkinson disease. Neurology. 2005;65(8):1239-45. http://dx.doi.org/10.1212/01. wnl.0000180516.69442.95

13. Factor SA, Feustel PJ, Friedman JH, Comella CL, Goetz CG, Kurlan R, et al. Longitudinal outcome of Parkinson's disease patients with psychosis. Neurology. 2003;60(11):1756-61. http://dx.doi. org/10.1212/01.WNL.0000068010.82167.CF

14. Goldstein DS, Sewell L, Sharabi Y. Autonomic dysfunction in PD: A window to early detection? J Neurol Sci. 2011;310(1-2):118-22. http://dx.doi.org/10.1016/j.jns.2011.04.011

15. Schrag A, Jahanshahi M, Quinn N. What contributes to quality of life in patients with Parkinson's disease? J Neurol, Neurosurg, Psychiatr. 2000;69(3):308-12. http://dx.doi.org/10.1136/jnnp.69.3.308

16. Williams-Gray CH, Mason SL, Evans JR, Foltynie T, Brayne C, Robbins TW, et al. The CamPaIGN study of Parkinson's disease: 10-year outlook in an incident population-based cohort. J Neurol, Neurosurg, Psychiatr. 2013;84(11):1258-64. http://dx.doi.org/10.1136/jnnp-2013-305277

17. Okuma Y, Yanagisawa N. The clinical spectrum of freezing of gait in Parkinson's disease. Mov Disord: Official J Mov Disord Soc. 2008;23 Suppl 2:S426-30. http://dx.doi.org/10.1002/mds.21934

18. Schrag A, Quinn N. Dyskinesias and motor fluctuations in Parkinson's disease. A community-based study. Brain: A J Neurol. 2000;123(Pt 11):2297-305.

19. McKeith IG, Boeve BF, Dickson DW, Halliday G, Taylor J-P, Weintraub D, et al. Diagnosis and management of dementia with Lewy bodies: Fourth consensus report of the DLB Consortium. Neurology. 2017;89(1):88-100. http://dx.doi.org/10.1212/WNL.0000000000004058

20. McKeith I, Del Ser T, Spano P, Emre M, Wesnes K, Anand R, et al. Efficacy of rivastigmine in dementia with Lewy bodies: A randomised, double-blind, placebo-controlled international 
study. Lancet (London, England). 2000;356(9247):2031-6. http://dx.doi.org/10.1016/ S0140-6736(00)03399-7

21. Schrag A, Ben-Shlomo Y, Quinn NP. Prevalence of progressive supranuclear palsy and multiple system atrophy: A cross-sectional study. The Lancet. 1999;354(9192):1771-5. http://dx.doi.org/10.1016/ S0140-6736(99)04137-9

22. Rehman HU. Multiple system atrophy. Postgraduate Med J. 2001;77(908):379-82. http://dx.doi. org/10.1136/pmj.77.908.379

23. Gilman S, Wenning GK, Low PA, Brooks DJ, Mathias CJ, Trojanowski JQ, et al. Second consensus statement on the diagnosis of multiple system atrophy. Neurology. 2008;71(9):670-6. http://dx.doi. org/10.1212/01.wnl.0000324625.00404.15

24. Ling H. Clinical approach to progressive Supranuclear Palsy. J Mov Disord. 2016;9(1):3-13. http:// dx.doi.org/10.14802/jmd.15060

25. Hoglinger GU, Respondek G, Stamelou M, Kurz C, Josephs KA, Lang AE, et al. Clinical diagnosis of progressive supranuclear palsy: The movement disorder society criteria. Mov Disord: Official J Mov Disord Soc. 2017;32(6):853-64. http://dx.doi.org/10.1002/mds.26987

26. Burrell JR, Hodges JR, Rowe JB. Cognition in corticobasal syndrome and progressive supranuclear palsy: A review. Mov Disord: Official J Mov Disord Soc. 2014;29(5):684-93. http://dx.doi. org/10.1002/mds.25872

27. Whitwell JL, Hoglinger GU, Antonini A, Bordelon Y, Boxer AL, Colosimo C, et al. Radiological biomarkers for diagnosis in PSP: Where are we and where do we need to be? Mov Disord: Official J Mov Disord Soc. 2017;32(7):955-71. http://dx.doi.org/10.1002/mds.27038

28. Armstrong MJ, Litvan I, Lang AE, Bak TH, Bhatia KP, Borroni B, et al. Criteria for the diagnosis of corticobasal degeneration. Neurology. 2013;80(5):496-503. http://dx.doi.org/10.1212/ WNL.0b013e31827fofdl

29. Koyama M, Yagishita A, Nakata Y, Hayashi M, Bandoh M, Mizutani T. Imaging of corticobasal degeneration syndrome. Neuroradiology. 2007;49(11):905-12. http://dx.doi.org/10.1007/ s00234-007-0265-6

30. Shin HW, Chung SJ. Drug-induced parkinsonism. Journal of clinical neurology (Seoul, Korea). 2012;8(1):15-21. http://dx.doi.org/10.3988/jcn.2012.8.1.15

31. Goldman SM. Environmental toxins and Parkinson's Disease. Ann Rev Pharmacol Toxicol. 2014;54(1):141-64. http://dx.doi.org/10.1146/annurev-pharmtox-011613-135937

32. Heim B, Krismer F, De Marzi R, Seppi K. Magnetic resonance imaging for the diagnosis of Parkinson's disease. J Neural Transm. 2017;124(8):915-64. http://dx.doi.org/10.1007/s00702-017-1717-8

33. Choi IS. Parkinsonism after carbon monoxide poisoning. Eur Neurol. 2002;48(1):30-3. http://dx.doi. org/10.1159/000064954

34. Thanvi B, Lo N, Robinson T. Vascular parkinsonism--an important cause of parkinsonism in older people. Age Ageing. 2005;34(2):114-9. http://dx.doi.org/10.1093/ageing/afi025

35. Glass PG, Lees AJ, Bacellar A, Zijlmans J, Katzenschlager R, Silveira-Moriyama L. The clinical features of pathologically confirmed vascular parkinsonism. J Neurol, Neurosurg, Psychiatr. 2012;83(10): 1027-9. http://dx.doi.org/10.1136/jnnp-2012-302828

36. Williams MA, Relkin NR. Diagnosis and management of idiopathic normal-pressure hydrocephalus. Neurol Clin Pract. 2013;3(5):375-85. http://dx.doi.org/10.1212/CPJ.0b013e3182a78f6b

37. Molde K, Soderstrom L, Laurell K. Parkinsonian symptoms in normal pressure hydrocephalus: A population-based study. J Neurol. 2017;264(10):2141-8. http://dx.doi.org/10.1007/s00415-0178598-5

38. Klinge P, Hellstrom P, TansJ, Wikkelso C. One-year outcome in the European multicentre study on iNPH. Acta Neurol Scand. 2012;126(3):145-53. http://dx.doi.org/10.111l/j.1600-0404.2012.01676.x

39. Bonifati V. Genetics of Parkinson's disease--state of the art, 2013. Parkinsonism Relat Disord. 2014;20 Suppl 1:S23-8. http://dx.doi.org/10.1016/S1353-8020(13)70009-9

40. Bekris LM, Mata IF, Zabetian CP. The genetics of Parkinson disease. J Geriatr Psychiatr Neurol. 2010;23(4):228-42. http://dx.doi.org/10.1177/0891988710383572

41. Healy DG, Falchi M, O'Sullivan SS, Bonifati V, Durr A, Bressman S, et al. Phenotype, genotype, and worldwide genetic penetrance of LRRK2-associated Parkinson's disease: A case-control study. The Lancet Neurol. 2008;7(7):583-90. http://dx.doi.org/10.1016/S1474-4422(08)70117-0 
42. Lorincz MT. Neurologic Wilson's disease. Ann N Y Acad Sci. 2010;1184:173-87. http://dx.doi. org/10.1111/j.1749-6632.2009.05109.x

43. Ruocco HH, Lopes-Cendes I, Laurito TL, Li LM, Cendes F. Clinical presentation of juvenile Huntington disease. Arquivos Neuro-Psiquiatr. 2006;64(1):5-9. http://dx.doi.org/10.1590/ S0004-282X2006000100002

44. Martino D, Stamelou M, Bhatia KP. The differential diagnosis of Huntington's disease-like syndromes: 'red flags' for the clinician. J Neurol, Neurosurg \& Psychiatr. 2013;84(6):650-6. http://dx.doi. org/10.1136/jnnp-2012-302532

45. Park H, Kim HJ, Jeon BS. Parkinsonism in spinocerebellar ataxia. BioMed Res Int. 2015;2015:125273. http://dx.doi.org/10.1155/2015/125273

46. Paulson H. Machado-Joseph Disease/Spinocerebellar Ataxia Type 3. Handbook of Clinical Neurology / edited by PJ Vinken and GW Bruyn. 2012;103:437-49. http://dx.doi.org/10.1016/ B978-0-444-51892-7.00027-9

47. Niu Y-Q, Yang J-C, Hall DA, Leehey MA, Tassone F, Olichney JM, et al. Parkinsonism in fragile X-associated tremor/ataxia syndrome (FXTAS): Revisited. Parkinsonism Relat Disord. 2014;20(4):4569. http://dx.doi.org/10.1016/j.parkreldis.2014.01.006

48. Siuda J, Fujioka S, Wszolek ZK. Parkinsonian syndrome in familial frontotemporal dementia. Parkinsonism Relat Disord. 2014;20(9):957-64. http://dx.doi.org/10.1016/j.parkreldis.2014.06.004

49. Schneider SA, Hardy J, Bhatia KP. Syndromes of neurodegeneration with brain iron accumulation (NBIA): An update on clinical presentations, histological and genetic underpinnings, and treatment considerations. Mov Disord: Official J Mov Disord Soc. 2012;27(1):42-53. http://dx.doi.org/10.1002/ mds. 23971

50. Puschmann A. Monogenic Parkinson's disease and parkinsonism: Clinical phenotypes and frequencies of known mutations. Parkinsonism Relat Disord. 2013;19(4):407-15. http://dx.doi.org/10.1016/j. parkreldis.2013.01.020

51. Davidzon G, Greene P, Mancuso M, Klos KJ, Ahlskog JE, Hirano M, et al. Early-onset familial parkinsonism due to POLG mutations. Ann Neurol. 2006;59(5):859-62. http://dx.doi.org/10.1002/ ana.20831

52. Nicolas G, Pottier C, Charbonnier C, Guyant-Marechal L, Le Ber I, Pariente J, et al. Phenotypic spectrum of probable and genetically-confirmed idiopathic basal ganglia calcification. Brain: J Neurol. 2013;136(Pt 11):3395-407. http://dx.doi.org/10.1093/brain/awt255

53. Elble RJ. The essential tremor syndromes. Curr Opin Neurol. 2016;29(4):507-12. http://dx.doi. org/10.1097/WCO.0000000000000347

54. Albanese A, Bhatia K, Bressman SB, Delong MR, Fahn S, Fung VS, et al. Phenomenology and classification of dystonia: A consensus update. Mov Disord: Official J Mov Disord Soc. 2013;28(7):863-73. http://dx.doi.org/10.1002/mds.25475

55. O'Sullivan SS, Williams DR, Gallagher DA, Massey LA, Silveira-Moriyama L, Lees AJ. Nonmotor symptoms as presenting complaints in Parkinson's disease: A clinicopathological study. Mov Disord: Official J Mov Disord Soc. 2008;23(1):101-6. http://dx.doi.org/10.1002/mds.21813

56. Williams DR, Lees AJ. How do patients with parkinsonism present? A clinicopathological study. Int Med J. 2009;39(1):7-12. http://dx.doi.org/10.1111/j.1445-5994.2008.01635.x

57. Kim YE, Lee WW, Yun JY, Yang HJ, Kim HJ, Jeon BS. Musculoskeletal problems in Parkinson's disease: Neglected issues. Parkinsonism Relat Disord. 2013;19(7):666-9. http://dx.doi.org/10.1016/j. parkreldis.2013.03.002

58. Organization WH. The ICD-10 classification of mental and behavioural disorders: Diagnostic criteria for research. Geneva: World Health Organization; 1993.

59. Christos G, Panagiotis K, Maria C, Roberto E, Bettina B, Gary P, et al. Revisiting the syndrome of "Obsessional Slowness". Mov Disord Clin Pract. 2015;2(2):163-9. http://dx.doi.org/10.1002/ mdc3. 12140

60. Jankovic J. Diagnosis and treatment of psychogenic parkinsonism. J Neurol, Neurosurg, Psychiatr. 2011;82(12):1300-3. http://dx.doi.org/10.1136/jnnp-2011-300876

61. Gowers WR. A manual of diseases of the central nervous system. Vol. II. London: J\&A Churchill; 1886. 